\title{
Enhancing Activity of Anticancer Drugs in Multidrug Resistant Tumors by Modulating P-Glycoprotein through Dietary Nutraceuticals
}

\author{
Muhammad Khan*,Amara Maryam, Tahir Mehmood, Yaofang Zhang, Tonghui Ma
}

\begin{abstract}
Multidrug resistance is a principal mechanism by which tumors become resistant to structurally and functionally unrelated anticancer drugs. Resistance to chemotherapy has been correlated with overexpression of p-glycoprotein (p-gp), a member of the ATP-binding cassette (ABC) superfamily of membrane transporters. P-gp mediates resistance to a broad-spectrum of anticancer drugs including doxorubicin, taxol, and vinca alkaloids by actively expelling the drugs from cells. Use of specific inhibitors/blocker of $p$-gp in combination with clinically important anticancer drugs has emerged as a new paradigm for overcoming multidrug resistance. The aim of this paper is to review p-gp regulation by dietary nutraceuticals and to correlate this dietary nutraceutical induced-modulation of $p$-gp with activity of anticancer drugs.
\end{abstract}

Keywords: Multidrug resistance - p-glycoprotein - dietary nutraceuticals - anticancer drugs

Asian Pac J Cancer Prev, 16 (16), 6831-6839

\section{Introduction}

Cancer is one of the most virulent, devastating and deadliest disease in the world and it represents the second leading cause of death worldwide (Jemal et al., 2008; Siegel et al., 2012), with approximately 14 million new cases and 8.2 million cancer related deaths in 2012 (Ferlay et al., 2015). Chemotherapy is one of the most effective treatments for metastatic cancers (Chang, 2010). Multidrug resistance (MDR) is one of the major obstacles in the successful chemotherapy of various cancers (Baguley, 2010; Eid et al., 2012; Xu et al., 2012; Kibria et al., 2014; Liu et al., 2014). It is widely accepted now that MDR exists against every effective drug (Callaghan et al., 2014). Therefore, the modulation of cellular molecules involved in MDR and circumvent drug resistance is likely to improve chemotherapy (Gottesman et al., 2002; Gillet et al., 2010).

P-glycoprotein, a $170 \mathrm{kDa}$ transmembrane phosphorylated glycoprotein encoded by MDR1 (ABCB1), belongs to ATP-binding cassette (ABC) superfamily of membrane transporters (Eichhorn and Efferth, 2012; Eid et al., 2012). It is composed of 1280 amino acids and consists of two similar halves each containing six transmembrane domains and one ATP binding domain (Nabekura, 2010a; Zeino et al., 2015). P-gp was first discovered in 1976 in Chinese hamster ovary $(\mathrm{CHO})$ cells, where it was found to display resistance to anticancer drugs (Callaghan et al., 2014). By the 1980s antibodies had been developed to $\mathrm{p}$-gp to detect the distribution and expression of this protein in various cells. Soon, it became evident that p-gp expressed in various cancer as well as numerous normal cells (Callaghan et al., 2014). In 1987, it was first detected in human KB carcinoma cells where its overexpression was found to be associated with cellular resistance to a wide range of anticancer drugs including colchincine, vinblastine, and doxorubicin (Ueda et al., 1987). Since then, researchers have compiled a myriad of research reports on the key role of p-gp in MDR (Bhardwaj et al., 2002; Eid et al., 2013; Molnar et al., 2010; Nabekura et al., 2008a; Nabekura et al., 2008b; Perestelo et al., 2011; Yang et al., 2014). It has become an established fact now that p-gp mediates MDR by actively transporting a wide range of anticancer drugs including paclitaxel, doxorubicin, daunorubicin, vinca alkaloids and eteposide (Zhang et al., 2003; Yoshida et al., 2005; Ferreira et al., 2006; Nabekura, 2010a; Yang et al., 2014). P-gp can interact and bind with a large number of structurally and functionally unrelated agents which suggests its multiple binding sites (Zhang et al., 2009; Chen et al., 2012). According to the interactions, these agents can be classified into three main groups: substrate, inhibitors, and modulators. Agents that are actively transported by p-gp are categorized as substrates whereas those that inhibit the transporting activity of $\mathrm{p}-\mathrm{gp}$ are termed as inhibitors. Modulators interact and bind with allosteric site of $\mathrm{p}$-gp thereby reducing the substrate binding through conformational changes. Thus, inhibitors and modulators exert the same biological effect (Chen et al., 2012). Therefore, identification and characterization of $\mathrm{p}$-gp inhibitors and modulators may provide a useful

${ }^{1}$ College of Basic Medical Sciences, Dalian Medical University, Dalian, Liaoning, P.R. China *For correspondence: khan2014@ dlmedu.edu.cn 
strategy for circumventing multidrug resistance.

Owing the importance of $\mathrm{p}$-gp on MDR, extensive studies have been conducted to identify p-gp inhibitors (Polli et al., 2001; Chen et al., 2012; Chang et al., 2006). A large number of p-gp inhibitors including verapamil and cyclosporine were identified. However, due to severe side effects or ineffectiveness, these inhibitors fail to achieve successful outcomes at clinical trials (Szakacs et al., 2006; Nabekura, 2010a). Generally speaking, dietary components are considered safe or less toxic to body than pharmaceutical drugs (Nabekura, 2010a; Mukkavilli et al., 2014). Fruits and vegetables are not only an excellent source of fiber, vitamins and minerals but also contain various components that may offer substantial health benefits beyond basic nutrition (Aggarwal et al., 2009; Nabekura, 2010a). Recent research has shown that more consumption of vegetables and fruits can prevent $20 \%$ incident of cancer and about 200,000 cancer related deaths annually (Pratheeshkumar et al., 2012). Other studies have shown that phytochemicals derived from fruits, vegetables, and spices have a great potential to enhance sensitivity of cancer cells to anticancer drugs (Borska et al., 2012; Ye et al., 2012; Kim et al., 2014). A food or a part of food that provide medical and health benefits including the prevention and/or treatment of a disease is called as Nutraceutical (Aggarwal et al., 2009). The acceptance and growing interest of dietary nutraceuticals as complementary medicines has further fuel opportunities for discovering interactions between p-gp and dietary nutraceuticals. In last three decades, a plethora of studies have been carried out to identify $\mathrm{p}$-gp inhibitors from dietary sources. The quest for $\mathrm{p}$-gp inhibitors from dietary sources has uncovered a long list of compounds (Zhou et al., 2004; Mahringer et al., 2010; Abdallah et al., 2015). In this review, we have selected only those nutraceuticals which have been shown to enhance the activity of known anticancer drugs through p-gp regulation. The chemical structures and dietary sources of these nutraceuticals have been presented in Figure 1-2.

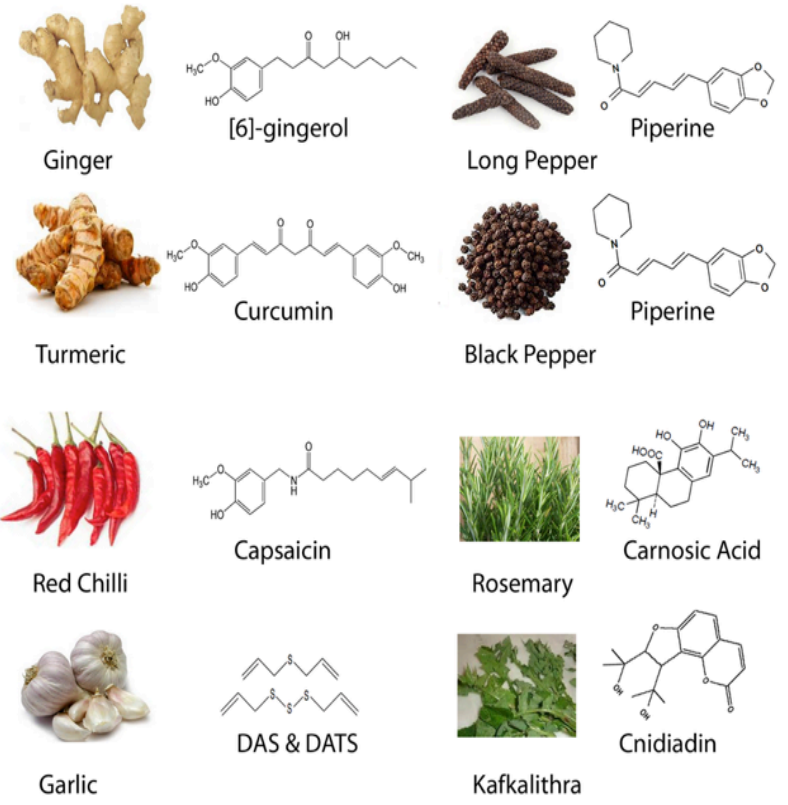

Figure 1. Chemical Structure and Source of Nutraceuticals Derived from Spices

\section{P-gp inhibitors and chemosensitizers of dietary origin}

Due to severe side effects and limited therapeutic success of synthetic drugs/chemotherapy against p-gpmediated MDR, some researchers have started to think over natural compounds of dietary origin as better alternatives. Over the past few decades, a substantial research has been dedicated to investigate the therapeutic benefit of the dietary components against p-gp-mediated MDR. A plethora of compounds and food extracts have been identified with varying effects on $\mathrm{p}$-gp regulation and reversal of MDR. However, our review will focus only on those promising nutraceuticals isolated from four major food groups; spices, fruits, vegetables and tea that not only inhibit p-gp but also enhance the sensitivity of tumor cells to anticancer drugs.

\section{Spices}

Spices have been amongst the most precious commodities since the time immemorial. The search of spices led to the great age of exploration and discovery of the new world, for example they led such legendary explorers as Christopher Columbus and Vasco de Gama to search the world for pepper and other spices (Aggarwal et al., 2009) Spices have been used for thousands of years in a wide variety of cultures as flavor agents, as colorants to add special taste to dishes, and also as a preservative to prevent

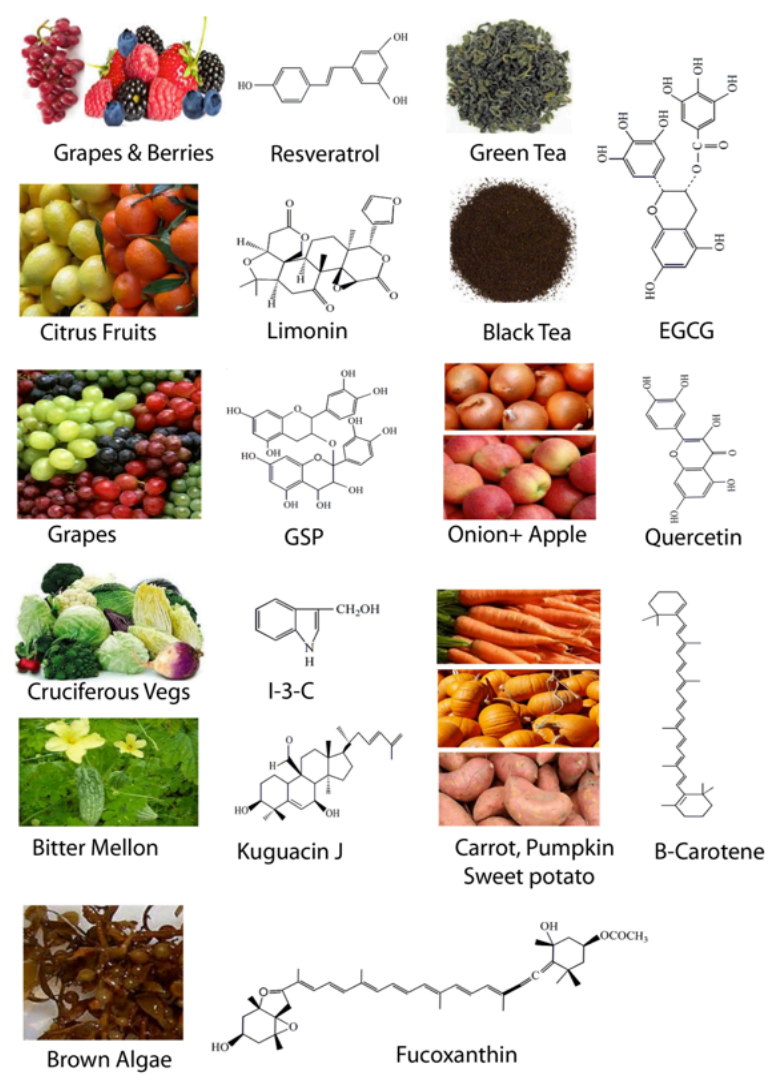

Figure 2. Chemical Structure and Source of Nutraceuticals Derived from Fruits, Vegetables and Tea 
Enhancing Anticancer Drug Activity in Multidrug Resistant Tumors by Modulating P-Glycoprotein with Dietary Nutraceuticals

the growth of bacteria. With the advancement of science, spices have been explored for their medicinal values. It is well established now that spice-derived photochemicals play a key role in the prevention and treatment of cancer by interfering with multiple mechanisms which are central to cancer progression (Aggarwal et al., 2008).

\section{Curcumin}

Curcumin is a major component of turmeric (Curcuma longa Linn). The content of curcumin in turmeric is about 1-5\%. Curcumin has been shown to exhibit a wide range of biological and pharmacological activities including antioxidant, anti-inflammatory, anti-infection and anticancer properties (Anuchapreeda et al., 2002). Recently, it has been investigated for its effects on $\mathrm{p}$-gp regulation in MDR tumor cells. Increaing lines of evidence suggested that curcumin reverse MDR through p-gp inhibition. Choi et al., (2008) investigated that curcumin inhibits the p-gp efflux activity through the inhibition of the PI3K/Akt/ NF-kB signaling pathway in multidrug resistant mouse leukemia L1210 cells. To address whether this p-gp inhibition by curcumin could sensitize multidrug resistant L1210 cells to chemotherapy, cells were treated with adriamycin and curcumin either alone or in combination of both and cleavage of PARP was observed by Western blot. The data demonstrated that both adriamycin and curcumin did not induce PARP cleavage however, coadministration of adriamycin with varying concentrations of curcumin resulted in significant cleavage of PARP in a dose-dependent manner (Choi et al., 2008).

Curcumin has also inhibited p-gp in drug sensitive SKOV3 and drug resistant SKOV3TR human ovarian adenocarcinoma cells. SKOV3TR cells overexpress p-gp, the classic marker of MDR phenomenon. The $\mathrm{IC}_{50}$ value of paclitaxel against SKOV3TR cells $(2.9 \mu \mathrm{M})$ was found to be 300 fold higher than SKOV3 $(9.5 \mathrm{nM})$ which indicates

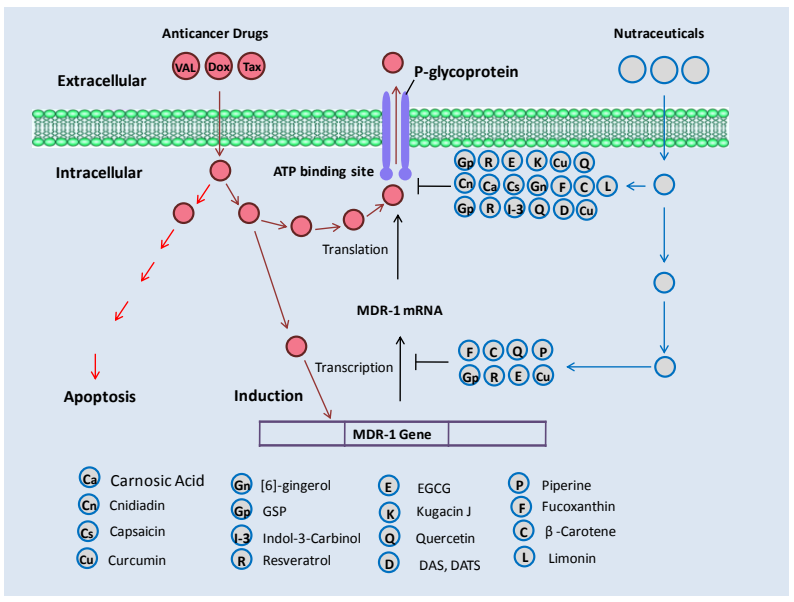

Figure 3. An illustration of How p-gp Modulators Can Inhibit the p-gp in MDR Tumor Cells and Enhance Sensitivity of Resistant Tumor Cells To Chemotherapy. Drug-induced or constitutive overexpression of p-gp actively transport anticancer drugs out of cells. P-gp modulators can inhibit the expression and function of p-gp, resulting in intracellular accumulation of anticancer drugs which then kill the cells by inducing apoptosis that paclitaxel is a substrate of p-gp. Co-administration of $5 \mu \mathrm{M}$ curcumin reduced the $\mathrm{IC}_{50}$ of paclitaxel from $2.9 \mu \mathrm{M}$ to 1.6 $\mu \mathrm{M}$ in SKOV3TR cells (Ganta and Amiji., 2009).

In A549 lung adenocarcinoma cells, curcumin has been shown to reverse cisplatin resistance through HIF$1 \alpha$-mediated p-gp down-regulation (Ye et al., 2012). In multidrug resistant human cervical carcinoma KB-V1 cells, curcumin decreased the mRNA expression of MDR 1 and protein expression of p-gp in a dose- and time- dependent manner. The maximum inhibitory effect was detected when cells were exposed to curcumin for 72 h. At $72 \mathrm{~h}$ time exposure, curcumin inhibited the MDR1 mRNA and p-gp protein expressions even at very low concentration $(1-10 \mu \mathrm{M})$ in a dose-dependent manner. However, curcumin inhibited P-gp-mediated efflux of rhodamine 123 and enhanced cytotoxicity of vinblastine at short time exposure (1-2 h), indicating that curcumin exerts its effects by inhibiting ATPase activity and function of $\mathrm{p}$-gp in $\mathrm{KB}-\mathrm{V} 1$ cells by directly interacting with $\mathrm{p}$-gp (Anuchapreeda et al., 2002).

\section{Piperine}

Piperine is the major plant alkaloids present in black pepper (Piper nigrum Linn) (Han et al., 2008; Do et al., 2013; Singh et al., 2013) and long pepper (Piper longum Linn) (Bezerra et al., 2008). Black pepper is one of the most common spice consumed by a large number of populations worldwide. Piperine has been reported to enhance the activity of anticancer drug in various drug resistant cancer cells (Bezerra et al., 2008). Other studies have shown that piperine inhibits the efflux activity of p-gp and alters the clinical pharmacokinetic profile of several drugs which are p-gp substrate (Zhang et al., 2009). However, only one recent study by Li et al. (2011) has provided evidence that piperine sensitizes multidrug resistant tumors cells to chemotherapy through $\mathrm{ABC}$ transporter inhibition. It effectively sensitized drug resistant MCF-7 and A549 cancer cells to doxorubicin. It reduced the $\mathrm{IC}_{50}$ values of doxorubicin against MCF-7 and A549 cells from 40.5 and $4.43 \mu \mathrm{M}$ to 1.26 and $0.31 \mu \mathrm{M}$ respectively when co-administered with doxorubicin at $50 \mu \mathrm{M}$ concentration. It is noteworthy that piperine itself at this concentration was not toxic to these cell lines. In addition to doxorubicin, piperine also sensitized the drug resistant MCF-7 cells to mitoxantrone. The $\mathrm{IC}_{50}$ value of mitoxantrone against drug resistant $\mathrm{MCF}-7$ cells was $37.72 \mu \mathrm{M}$ which reduced to $5.41 \mu \mathrm{M}$ in the presence of 50 $\mu \mathrm{M}$ piperine. Moreover, piperine has inhibited the mRNA expression of MDR1, ABCC1 and ABCG2 which encode P-gp, MRP1 and BCRP1 respectively. Of note, piperine was found more effective than MK-571 (multidrug resistant protein 1, MRP1 inhibitor) in drug resistant A549 cells at equivalent concentration of inhibitor.

\section{Capsaicin}

Capsaicin is the principal pungent component of red chilli (Capsicum frutescens). Capsaicin has been shown to exhibit anticancer properties in various cancer cell lines in culture and animal models (Aggarwal et al., 2008). In 
addition to anticancer activity, it has been reported to increase intracellular accumulation of daunorubicin and rhodamine 123 by inhibiting the p-gp efflux activity in multidrug-resistant human carcinoma KB-C2 cells. It also increased the cellular accumulation of vinblastine and potentiated its anticancer activity in $\mathrm{KB}-\mathrm{C} 2$ cells (Nabekura et al., 2005). These findings indicate that capsaicin potentially inhibits $\mathrm{p}-\mathrm{gp}$ and potentiates vinblastine anticancer activity through $\mathrm{p}$-gp modulation.

\section{[6]-Gingerol}

[6]-gingerol is the major phenolic bioactive component extracted from rhizome of ginger (Zingiber officinale) which is responsible for spicy taste of ginger. It has been shown to exhibit antiproliferative activity against a wide range of cancer cells by inhibiting various survival pathways including NF-KB and $\beta$-catenine (Nabekura, 2010a). Like capsaicin, it has also increased intracellular accumulation of daunorubicin and rhodamine 123 by inhibiting the p-gp efflux activity in multidrug-resistant human carcinoma KB-C2 cells. It enhanced the toxicity of vinblastine in $\mathrm{KB}-\mathrm{C} 2$ cells through p-gp inhibition (Nabekura et al., 2005). However, the available published data is not sufficient to explain the exact mechanism of p-gp inhibition by [6]-gingerol.

\section{DAS (Diallyl sulfide) and DATS (Diallyl trisulfide)}

DAS and DATS are the volatile organosulfur compounds present in garlic (Allium sativum), which is one of the most widely used spice in the world (Zhang et al., 2009). Various studies have been conducted to observe the effect of organosulfur compounds of garlic on p-gp expression. A controversial data has been presented regarding $\mathrm{p}$-gp regulation by these components. For example, in colo 205 human colon cancer cells, DATS has been shown to induce MDR1 gene expression while DAS did not induce any effect on MDR1 gene expression (Lai et al., 2012). However, both DAS and DATS have been reported to reverse MDR of K562 leukemia cell lines (Arora et al., 2004; Xia et al., 2012). DAS increased the intracellular accumulation of doxorubicin in drug resistant K562/R10 cells at a concentration of $8.75 \mathrm{mM}$. DAS itself did not show any toxic effect on cell viability at this very high concentration. DAS, at this concentration not only potentiated the cytotoxicity of vinca alkaloids (Vinblastine and Vincristine) in drug resistant K562/R10 cells but also decreased the expression of p-gp significantly in timedependent manner. DAS also inhibited the vinblastineinduced expression of $\mathrm{p}$-gp in normal mouse liver (Arora et al., 2004).

Similar results have been presented for DATS by Xia et al. (2012) in drug resistant K562/A02 leukemia cells. In K562/A02 cells, DATS increased the cytotoxicity of daunorubicin by increasing its intracellular accumulation and decreasing $\mathrm{p}$-gp expression at $2 \mu \mathrm{M}$ concentration for $48 \mathrm{~h}$ (Xia et al., 2012). This incongruent data of DAS and DATS might be due to different experimental models or different cancer types.

\section{Carnosic Acid}

Carnosic acid, a phenolic diterpene is a major component of leaves of rosemary (Rosmarinus officinalis). Rosemary leaves are commonly used as spice in cooking. A typical commercial rosemary extract contains about $20 \%(w / w)$ carnosic acid. Recently, the effect of carnosic acid on $\mathrm{p}$-gp regulation has been investigated in KB-C2 cells. Carnosic acid inhibited the efflux of p-gp substrates (daunorubicin and rhodamine 123) in KB-C2 cells in a dose-dependent manner. Carnosic acid at $10 \mu \mathrm{M}$ reversed the p-gp-mediated MDR by sensitizing $\mathrm{KB}-\mathrm{C} 2$ cells to vinblastine. Like other known p-gp substrates such as verapamil, carnosic acid stimulated the ATPase activity of $\mathrm{p}$-gp which indicates that carnosic acid might be a substrate for $\mathrm{p}$-gp and competitively binds at substrate binding site of p-gp (Nabekura et al., 2010b).

\section{Cnidiadin}

Cnidiadin, a furanocoumarin compound is present in Tordylium apulum, a plant commonly used as spice in Greek cooking with the name Kafkalithra and one of the most aromatic greens for savory pie fillings. Cnidiadin at a concentration of $10 \mu \mathrm{M}$ has potentiated the cytotoxic effects of vinblastine and vincristine against multiresistant MDCK-MDR1 and epidermoid carcinoma KB cell lines respectively. Cumulative experimental data indicate that cnidiadin is a specific inhibitor of $\mathrm{p}$-gp and it sensitizes multidrug resistant tumor cells to chemotherapy by competitively binding and inhibiting the efflux of drug by p-gp (Barthomeuf et al., 2005).

\section{Fruits and Vegetables}

Increased consumption of fruits and vegetables can prevent various diseases and disorders. A large body of evidence from various case-control studies has shown that intake of fruits and vegetables have a strong protective effect against a wide variety of human cancers (Nile and Park, 2014; Temple and Gladwin, 2003). Recent research has shown that more consumption of vegetables and fruits can prevent $20 \%$ incident of cancer and about 200,000 cancer related deaths annually (Pratheeshkumar et al., 2012). Recently, a growing interest has been found to investigate the role of fruits and vegetables in reversing multidrug resistance. Several potent $\mathrm{p}$-gp inhibitors have been identified and their role in reversing multidrug resistance has been studies in tumor cell culture studies. The chemical structure and source of nutraceuticals derived from fruits and vegetables have been shown in Figure 2.

\section{Resveratrol}

Resveratrol is a polyphenolic phytoalexin produced in many plants in response to environmental stresses such as exposure to ozone, sunlight, heavy metals and infection by pathogens. It is abundantly present in red grapes, berries (raspberries, blueberries, and mulberries), Scots pine, Eastern white pine, and knotweed. The anticancer 
Enhancing Anticancer Drug Activity in Multidrug Resistant Tumors by Modulating P-Glycoprotein with Dietary Nutraceuticals

and chemopreventive effects of resveratrol have been well documented in many in vitro and in vivo studies (Jasinski et al., 2013). In last few years, researchers have found that in addition to anticancer activity, resveratrol has a great potential to reverse MDR of breast cancer and myeloid leukemia cells. It has been shown to increase the intracellular accumulation and anticancer activity of doxorubicin in doxorubicin resistant MCF-7 breast carcinoma cells at $12 \mu \mathrm{M}$ concentration. This reversal effect of resveratrol on MDR appeared to result from down-regulation of MDR1 mRNA expression and p-gp protein expression (Huang et al., 2014). The reversal effect of resveratrol on multidrug resistant MCF-7 breast cancer cells have recently been published by another group who showed for the first time that resveratrol not only overcome multidrug resistance in vitro cell studies but also exhibit similar effects in vivo xenograft model. These effects appear to come from down-regulated expressions of MDR1 and MRP1 at mRNA as well as at protein level and reduced p-gp ATPase activity (Kim et al., 2014). Resveratrol has also been shown to reverse drug resistance phenotype of doxorubicin resistant AML-2 myeloid leukemia cells via down-regulation of the expression level and activity of MRP1 transporter molecule (Kweon et al., 2010).

\section{GSP (Grape seed procyanidin)}

GSP,a class of polyphenolic compounds, is found in high concentration in grape seeds as well as in many other fruits, vegetables and tea leaves (Chen et al., 2013). GSP is a nutritional supplement with known disease prevention characteristics. It has both chemopreventive and antiproliferative effects on a wide range of cancer cell lines, indicating a potential for application in cancer management (Hsu et al., 2009).

Recently, it has been found that catechin-3-O-2leucocyanidin a GSP, could reverse cancer cells MDR by modulating the expression and activity of p-gp. GSP has been shown to sensitize paclitaxel resistant A2780 human ovarian cancer cells to paclitaxel and adrimycin. This effect was found to be associated with p-gp inhibition. Further mechanistic study revealed that GSP reversed MDR by inhibiting the function and expression of $\mathrm{p}-\mathrm{gp}$ via inhibition of NF-KB and YB-1 translocation into nucleus through dephosphorylation of AKT and ERK1/2 respectively (Zhao et al., 2013).

\section{Limonin}

Limonin, a limonoid, is a white crystalline substance abundantly found in citrus fruits and contributes to the bitter taste of some citrus food products. Limonoid are considered as one of the most important components of medical food (Mahmoud et al., 2014). Recently, limonin has been studied for its effects on p-gp. It has been shown to inhibit p-gp in human melanoma, colon and leukemia cell lines. In WM-266-4 melanoma cells, it inhibited p-gp (Han et al., 2011) while in Caco-2 colon and CEM/ ADR5000 leukemia cells, it increased the intracellular accumulation of Rhodamine 123 and doxorubicin.
Moreover, limonin effectively enhanced the anticancer activity of doxorubicin in drug resistant Caco- 2 colon and CEM/ADR5000 leukemia cells at a concentration of $20 \mu \mathrm{M}$.

\section{Quercetin}

Quercetin is one of the most important flavonoid with well recognized multiple biological and pharmacological properties. It is present in many fruits and vegetables (Borska et al., 2012). It is most abundantly found in onion and apple. Apple skin, white onion bulb and onion leaves contain 250,50 , and $841 \mathrm{mg} / \mathrm{kg}$ quercetin respectively (Wach et al., 2007). Quercetin has been found to inhibit p-gp activity and expression in different experimental models (Zhou and Lim, 2004; Chieli et al., 2010). Recently, Quercetin has been reported as a good chemosensitizer. It has been shown to sensitize EPG85-257 drug resistant human gastric cancer cells to daunorubicin through inhibiting MDR1 and P-gp expression and its transport activity (Borska et al., 2012). Li et al., (2013) found that quercetin could enhance the anticancer activity of doxorubicin against multidrug resistant MCF-7 breast carcinoma by inhibiting the expression and activity of p-gp at a very low concentration of $0.7 \mu \mathrm{M}$.

\section{$\beta$-Carotene}

$\beta$-Carotene, a precursor of vitamin A, belongs to the group of naturally occurring carotenoids and is found in many fruits and vegetables including carrot, pumpkin, and sweet potato (Vrolijk et al., 2015). The pronounced and versatile biological and pharmacological effects have centered the attention of $\beta$-Carotene in cancer therapy. The MDR reversal effect of $\beta$-Carotene has recently been described. $\beta$-Carotene has accumulated Rhodamine 123, calcein AM, and doxorubicin in Caco-2 cell dose-dependently. It also stimulated the efficacy of 5-Fluorouracil, doxorubicin, and eteposide in Caco-2 cells. Its impact on resistance mechanism involved downregulation of MDR1 gene expression and inhibition of p-gp transport activity in Caco-2 cells (Eid et al., 2012).

\section{Indol-3-Carbinol (I-3-C)}

I-3-C is present in cruciferous vegetables such as broccoli, cauliflower, cabbage, Chinese cabbage, radishes, daikon, and turnip (Aggarwal and Ichikawa, 2005). It is active against a wide variety of tumors (Aggarwal and Ichikawa, 2005; Zhang et al., 2009). The capacity of I-3-C and its acid condensation derivatives to reverse MDR has been investigated in doxorubicin and vinblastine resistant B16/hMDR1 cells (melanoma cells transfected with human MDR1 gene) (Christensen and LeBlanc, 1996). In cell culture model, I-3-C acid condensation derivatives but not I-3-C enhanced the response of B16/hMDR1 cells to doxorubicin and vinblastine. The acid condensation derivatives competed with azidopine for binding with p-gp, indicating the direct interaction of these derivatives with p-gp. Co-administration of 333 or $500 \mathrm{mg} / \mathrm{kg}$ mouse/ day of I-3-C with doxorubicin or vinblastine significantly 
reduced the tumor size compared to either drug or I-3-C alone. This effect seems to come from conversion of I-3-C into its acid condensation derivatives in vivo which sensitize the tumor to doxorubicin or vinblastine.

In vinblastine resistant K562 human leukemia cells, I-3-C enhanced the response of cells to vinblastine at a non-toxic concentration of $10 \mathrm{mM}$ through downregulating the expression of $\mathrm{p}$-gp in a time-dependent fashion (Arora et al., 2005). Reversal of vinca alkaloidinduced overexpression of p-gp in mice at a dose of $5 \mathrm{mg}$ / $\mathrm{kg}$ has also been reported by the same research group (Arora and Shukla, 2003). However, it remains to be determined whether dietary intake of such vegetables contains that high amount of I-3-C to inhibit p-gp expression and activity in human body.

\section{Kuguacin J}

Kuguacin $\mathbf{J}$, a triterpenoid is a component of bitter mellon (Momordica charantia) which is a culinary bitter vegetable widely used in traditional dishes and in folk medicine in Asian countries (Sagor et al., 2015). Extracts of bitter melon have been reported to possess anticancer activity. The MDR reversing property of Kuguacin J has recently been investigated in human cervical carcinoma KB-V1 cells (Pitchakarn et al., 2012). It increases the intracellular accumulation of rhodamine 123 and calcein by inhibiting the efflux activity of p-gp in KB-V1 cells. Co-administration of 5 and $10 \mu \mathrm{M}$ of kuguacin $\mathrm{J}$ enhanced the response of KB-V1 cells to vinblastine and paclitaxel, 1.9- and 4.3-fold respectively, for vinblastine, and 1.9- and 3.2-fold respectively, for paclitaxel. The experimental data demonstrated that kuguacin $\mathrm{J}$ inhibits the transport activity of p-gp by directly interacting with substratebinding site of $\mathrm{p}-\mathrm{gp}$.

\section{Fucoxanthin}

Fucoxanthin, a carotenoid with widespread biological and pharmacological activities, is abundantly found in edible brown seaweeds (Brown algae) such as wakame (Undaria pinnatifida), kombu (Laminaria japonica), and hijiki (Sargassum fusiforme) which are staples in East Asians diet, especially in Japan and Korea (Zorofchian et al., 2014).

The MDR reversal effect of fucoxanthin has recently been reported. Fucoxanthin has stimulated intracellular accumulation of Rhodamine 123, calcein AM, and doxorubicin in Caco-2 cell in a dose-dependent manner. It significantly improved the efficacy of 5-Fluorouracil, vinblastine and eteposide in Caco-2 cells. Its impact on resistance mechanism involved down-regulation of MDR1 gene expression and inhibition of p-gp transport activity in Caco-2 cells (Eid et al., 2012).

\section{Tea}

Tea is one of the most popular and widely consumed flavored and functional drinks worldwide. Green and black tea are the most popular which account for about $20 \%$ and $78 \%$ of world consumption respectively. A large body of scientific reports has provided convincing health promoting evidence of tea consumption ( $\mathrm{Li}$, et al., 2013). Green tea extracts have been reported to possess chemopreventive and anticancer effects in a variety of human cancer cells through multiple mechanisms ( $\mathrm{Li}$, et al., 2013). Various components of green tea have been reported to inhibit transporter activity of $\mathrm{p}$-gp in various cancer cells (Zhang et al., 2009) however; our focus in this review is EGCG which possesses MDR reversal effects through p-gp regulation.

\section{Epigallocatechin gallate (EGCG)}

EGCG is a major water extractable component of green (Nabekura, 2010a; Li, et al., 2013) and black tea (Li, et al., 2013). A cup of green tea (2.5 g dried leaves) may contain about $90 \mathrm{mg}$ of EGCG (Li, et al., 2013). EGCG potentiated the cytotoxicity of vinblastine and doxorubicin against the Chinese hamster ovary resistant Cells CHRC5 (Jodoin et al., 2002) and the human oral epidermoid MDR KB-A-1 cells (Mei et al., 2004; Qian et al., 2005), respectively. Co-administration of EGCG $(40 \mathrm{mg} / \mathrm{kg})$ and doxorubicin $(2 \mathrm{mg} / \mathrm{kg})$ significantly inhibited the growth of tumor in KB-A-1 cells bearing nude mice xenograft. The MDR reversal activity of EGCG appeared to result from modulation of $\mathrm{p}$-gp function as MDR1 mRNA expression remained unchanged during this study (Qian et al., 2005). Contrasting results have been presented by Mei et al. (2004) who showed downregulation of MDR1 mRNA expression in KB-A-1 cells after 72 treatment of EGCG. However, EGCG increased intracellular accumulation of doxorubicin within $3 \mathrm{~h}$ in the same experimental model. This indicates that EGCG exerts reversal effect on MDR through modulation of p-gp transport activity. The study of Jodoin et al. (2002) also supports the notion that EGCG exerts MDR reversal effects through modulating the function of $\mathrm{p}$-gp rather than inhibition of p-gp expression.

\section{Conclusion and Future Perspectives}

In this review, we have summarized the recent progress on dietary nutraceuticals in reversing MDR through p-gp regulation in various in vitro and in vivo cancer models. Collective data from multitudinous studies have provided persuasive evidence that dietary nutraceuticals play an important role in overcoming MDR by modulating the expression and function of $\mathrm{p}$-gp through multiple mechanisms. They have been shown to modulate $\mathrm{p}$-gp by down-regulating MDR1 and p-gp expression or by directly binding with ATP-binding site, substrate binding site or allosteric site. However, inhibition of p-gp function to sensitize MDR phenotype of various cancers to chemotherapy was found to be major mechanism of dietary nutraceuticals mentioned in this review. It is important to note that majority of these nutraceuticals have only been evaluated for their MDR reversal potential in vitro or in vivo animal models. What are the effective doses of these nutraceuticals in human and whether these effective doses could be obtained by consuming fruits, vegetable, spices, and tea, are the questions that need to 
Enhancing Anticancer Drug Activity in Multidrug Resistant Tumors by Modulating P-Glycoprotein with Dietary Nutraceuticals be addressed.

In light of aforementioned findings, it can be speculated that given nutraceuticals may become potential lead compounds to overcome MDR in future. However, preclinical and clinical trials are yet required to elucidate the full spectrum of MDR reversal effects of these nutraceuticals in combination with other standard drugs to validate the further usefulness as potent MDR reversal agents.

\section{References}

Abdallah HM, Al-Abd AM, El-Dine RS, et al (2015). P-glycoprotein inhibitors of natural origin as potential tumor chemo-sensitizers: A review. J Adv Res, 6, 45-62.

Aggarwal BB, Ichikawa H, (2005). Molecular targets and anticancer potential of indole-3-carbinol and its derivatives. Cell Cycle, 4, 1201-15.

Aggarwal BB, Kunnumakkara AB, Harikumar KB, et al (2008). Potential of spicederived phytochemicals for cancer prevention. Planta Med, 74, 1560-9.

Aggarwal BB, Van Kuiken ME, Iyer LH, et al (2009). Molecular targets of nutraceuticals derived from dietary spices: potential role in suppression ofinflammation and tumorigenesis. Exp Biol Med, 234, 825-49.

Anuchapreeda S, Leechanachai P, Smith MM, et al (2002). Modulation of P-glycoprotein expression and function by curcumin in multidrug-resistant human KB cells. Biochem Pharmacol, 64, 573-82.

Arora A, Shukla Y (2003). Modulation of vinca-alkaloid induced P-glycoprotei expression by indole-3carbinol. Cancer Lett, 189, 167-73.

Arora A, Seth K, Shukla Y, (2004). Reversal of $\mathrm{P}$-glycoprotein-mediated multidrug resistance by diallyl sulfide in K562 leukemic cells and in mouse liver. Carcinogenesis, 25, 941-9.

Arora A, Seth K, Kalra N, et al (2005). Modulation of $\mathrm{P}$-glycoprotein-mediated multidrug resistance in K562 leukemic cells by indole-3-carbinol. Toxicol Appl Pharmacol, 202, 237-43.

Baguley BC, (2010). Multiple drug resistance mechanisms in cancer. Mol Biotechnol, 46, 308-16.

Barthomeuf C, Grassi J, Demeule M, et al (2005). Inhibition of P-glycoprotein transport function and reversion of MDR1 multidrug resistance by cnidiadin. Cancer Chemother Pharmacol, 56, 173-81.

Bezerra DP, de Castro FO, Alves AP, et al (2008). In vitro and in vivo antitumor effect of 5-FU combined with piplartine and piperine. J Appl Toxicol, 28, 156-63.

Bhardwaj RK, Glaeser H, Becquemont L,et al (2002). Piperine, a major constituent of black pepper, inhibits human P-glycoprotein and CYP3A4.J Pharmacol Exp Ther, 302, 645-50.

Borska S, Chmielewska M, Wysocka T, et al (2012). In vitro effect of quercetin on human gastric carcinoma: targeting cancer cells death and MDR. Food Chem Toxicol, 50, 3375-83.

Callaghan R, Luk F, Bebawy M, (2014). Inhibition of the multidrug resistance $\mathrm{P}$-glycoprotein: time for a change

of strategy? Drug Metab Dispos, 42, 623-31.

Chang C, Bahadduri PM, Polli JE, et al (2006). Rapid identification of P-glycoprotein substrates and inhibitors. Drug Metab Dispos, 34, 1976-84.

Chang XB, (2010). Molecular mechanism of ATPdependent solute transport by multidrug resistance associated protein 1. Methods Mol Biol, 596, 223-49

Chen L, Li Y, Yu H, et al (2012). Computational models for predicting substrates or inhibitors of P-glycoprotein. Drug Discov Today, 17, 343-51.

Chen Q, Zhang R, Li WM, et al (2013). The protective effect of grape seed procyanidin extract against cadmium-induced renal oxidative damage in mice. Environ Toxicol Pharmacol, 36, 759-68.

Chieli E, Romiti N, Rodeiro I, et al (2010). In vitro modulation of ABCB1/P-glycoprotein expression by polyphenols from Mangiferaindica. Chem Biol Interact, 186, 287-94.

Choi BH, Kim CG, Lim Y, et al (2008). Curcumin downregulates the multidrug-resistance mdr1b gene by inhibiting the PI3K/Akt/NF kappa B pathway. Cancer Lett, 259, 111-8.

Christensen JG, LeBlanc GA, (1996). Reversal of multidrug resistance in vivo by dietary administration of the phytochemical indole-3-carbinol. Cancer Res, 56, 574-581.

Do MT, Kim HG, Choi JH, et al (2013).Anti-tumor efficacy of piperine in the treatment of human HER2overexpressing breast cancer cells. Food Chem, 141, 2591-9.

Eichhorn T, Efferth T (2012). P-glycoprotein and its inhibition in tumors by phytochemicals derived from Chinese herbs. J Ethnopharmacol, 141, 557-70.

Eid SY,El-Readi MZ, Wink M (2012). Carotenoids reverse multidrug resistance in cancer cells by interfering with ABC-transporters. Phytomedicine, 19, 977-87.

Eid SY, El-Readi MZ, Eldin EE, et al (2013). Influence of combinations of digitonin with selected phenolics, terpenoids, and alkaloids on the expression and activity of P-glycoprotein in leukaemia and colon cancer cells. Phytomedicine, 21, 47-61.

Ferlay J, Soerjomataram I, Dikshit R, et al (2015). Cancer incidence and mortality worldwide: sources, methods and major patterns in GLOBOCAN 2012. Int J Cancer, 136, 359-86.

Ferreira MJ, Duarte N, Gyemant N, et al (2006). Interaction between doxorubicin and the resistance modifier stilbene on multidrug resistant mouse lymphoma and human breast cancer cells. Anticancer Res, 26, 3541-6.

Ganta S, Amiji M (2009). Coadministration of Paclitaxel and curcumin in nanoemulsion formulations to overcome multidrug resistance in tumor cells. $\mathrm{Mol}$ Pharm, 6, 928-39.

Gillet JP, Gottesman MM, (2010). Mechanisms of multidrug resistance in cancer. Methods Mol Biol, 596, 47-76.

Gottesman MM, Fojo T, Bates SE (2002). Multidrug resistance in cancer: role of ATP-dependent transporters. Nat Rev Cancer, 2, 48-58.

Han Y, Chin Tan TM, Lim LY (2008). In vitro and in vivo 
evaluation of the effects of piperine on P-gp function and expression. Toxicol Appl Pharmacol, 230, 283-9.

Han YL, Yu HL, Li D, et al (2011). Inhibitory effects of limonin on six human cytochrome P450 enzymes and P-glycoprotein in vitro. Toxicol In vitro, 25, 1828-33.

Hsu CP, Lin YH, Chou CC, et al (2009). Mechanisms of grape seed procyanidin-induced apoptosis in colorectal carcinoma cells. Anticancer Res, 29, 283-9.

Huang F, Wu XN, Chen J, et al (2014). Resveratrol reverses multidrug resistance in human breast cancer doxorubicin-resistant cells. Exp Ther Med, 7, 1611-6.

Jasinski M, Jasinska L, Ogrodowczyk M, (2013). Resveratrol in prostate diseases - a short review. Cent European J Urol, 66, 144-9.

Jemal A, Siegel R, Ward E, et al (2008). Cancer statistics, 2008. CA Cancer J Clin, 58, 71-96.

Jodoin J, Demeule M, Beliveau R (2002). Inhibition of the multidrug resistance P-glycoprotein activity by green tea polyphenols. Biochim Biophys Acta, 1542, 149-59.

Kibria G, Hatakeyama H, Harashima H, (2014). Cancer multidrug resistance: mechanisms involved and strategies for circumvention using a drug delivery system. Arch Pharm Res, 37, 4-15.

Kim TH, Shin YJ, Won AJ, et al (2014). Resveratrol enhances chemosensitivity of doxorubicin in multidrug-resistant human breast cancer cells via increased cellular influx of doxorubicin. Biochim Biophys Acta, 1840, 615-25.

Kweon SH, Song JH, Kim TS (2010). Resveratrolmediated reversal of doxorubicin resistance in acute myeloid leukemia cells via downregulation of MRP1 expression. Biochem Biophys Res Commun, 395, 104-10.

Lai KC, Kuo CL, Ho HC, et al (2012). Diallyl sulfide, diallyl disulfide and diallyltrisulfide affect drug resistant gene expression in colo 205 human colon cancer cells in vitro and in vivo. Phytomedicine, 19, 625-30.

Li S, Lei Y, Jia Y, et al (2011). Piperine, apiperidine alkaloid from Piper nigrum re-sensitizes P-gp, MRP1 and BCRP dependent multidrug resistant cancer cells. Phytomedicine, 19, 83-7.

Li S, Lo CY, Pan MH, et al (2013). Black tea: chemical analysis and stability. Food Funct, 4, 10-8.

Liu Z, Duan ZJ, Chang JY, et al (2014). Sinomenine sensitizes multidrug-resistant colon cancer cells (Caco-2) to doxorubicin by downregulation of MDR-1 expression. PLoS One, 9, 98560.

Mahmoud MF, Gamal S, El-Fayoumi HM (2014). Limonin attenuates hepatocellular injury following liver ischemia and reperfusion in rats via toll-like receptor dependent pathway. Eur J Pharmacol, 740, 676-82.

Mahringer A, Karamustafa S, Klotz D, et al (2010). Inhibition of P-glycoprotein at the blood-brain barrier by phytochemicals derived from traditional Chinese medicine. Cancer Genomics Proteomics, 7, 191-205.

Mei Y, Qian F, Wei D, et al (2004). Reversal of cancer multidrug resistance by green tea polyphenols. $J$ Pharm Pharmacol, 56, 1307-14.

Molnar J, Engi H, Hohmann J, et al (2010). Reversal of multidrug resitance by natural substances from plants. Curr Top Med Chem, 10, 1757-68.

Mukkavilli R, Gundala SR, Yang C, et al (2014). Modulation of cytochrome P450 metabolism and transport across intestinal epithelial barrier by ginger biophenolics. PLoS One, 9, 108386.

NabekuraT, Kamiyama S, Kitagawa S, (2005). Effects of dietary chemopreventive phytochemicals on P-glycoprotein function. Biochem Biophys Res Commun, 327, 866-70.

Nabekura T, Yamaki T, Kitagawa S (2008a). Effects of chemopreventive citrus phytochemicals on human P-glycoprotein and multidrug resistance protein 1 . Eur J Pharmacol, 600, 45-9.

Nabekura T, Yamaki T, Ueno K, et al (2008b). Inhibition of P-glycoprotein and multidrug resistance protein 1 by dietary phytochemicals. Cancer Chemother Pharmacol, 62, 867-73

Nabekura T (2010a). Overcoming multidrug resistance in human cancer cells by natural compounds. Toxins (Basel), 2, 1207-24.

Nabekura T, Yamaki T, Hiroi T, et al (2010b). Inhibition of anticancer drug efflux transporter P-glycoprotein by rosemary phytochemicals. Pharmacol Res, 61, 259-63.

Nile SH, Park SW, (2014). Edible berries: bioactive components and their effect on human health. Nutrition, 30, 134-44.

Perestelo NR, Sanchez-Canete MP, Gamarro F, et al (2011). Overcoming human P-glycoprotein-dependent multidrug resistance with novel dihydro-beta- agarofuransesquiterpenes. Eur J Med Chem, 46, 4915-23.

Pitchakarn P, Ohnuma S, Pintha K, et al (2012). Kuguacin $\mathrm{J}$ isolated from Momordica charantia leaves inhibits P-glycoprotein (ABCB 1)-mediated multidrug resistance. J Nutr Biochem, 23, 76-84.

Polli JW, Wring SA, Humphreys JE, et al (2001). Rational use of in vitro P-glycoprotein assays in drug discovery. J Pharmacol Exp Ther, 299, 620-8.

Pratheeshkumar P, Sreekala C, Zhang Z, et al (2012). Cancer prevention with promising natural products: mechanisms of action and molecular targets. Anticancer Agents Med Chem, 12, 1159-84.

Qian F, Wei D, Zhang Q, et al (2005). Modulation of P-glycoprotein function and reversal of multidrug resistance by (-)-epigallocatechingallate in human cancer cells. Biomed Pharmacother, 59, 64-9.

Sagor AT, Chowdhury MR, Tabassum N, et al (2015). Supplementation of fresh ucche (Momordicacharantia L. var. muricataWilld) prevented oxidative stress, fibrosis and hepatic damage in $\mathrm{CCl} 4$ treated rats. $B M C$ Complement Altern Med, 15, 115.

Siegel R, Naishadham D, Jemal A (2012). Cancer statistics for Hispanics/Latinos, 2012. CA Cancer J Clin, 62, 283-98.

Singh AK, Misra K, (2013). Human papilloma virus 16 E6 protein as a target for curcuminoids, curcumin conjugates and congeners for chemoprevention of oral and cervical cancers. Int Discip Sci, 5, 112-8.

Szakacs G, Paterson JK, Ludwig JA, et al (2006). Targeting multidrug resistance in cancer. Nat Rev Drug Discov, 5, 219-34. 
Temple NJ, Gladwin KK, (2003). Fruit, vegetables, and the prevention of cancer: research challenges. Nutrition, 19, 467-70.

Ueda K, Cardarelli C, Gottesman MM, et al (1987). Expression of a full-length cDNA for the human "MDR1" gene confers resistance to colchicine, doxorubicin, and vinblastine. Proc Natl Acad Sci USA, 84, 3004-8.

Vrolijk MF, Opperhuizen A, Jansen EH, et al (2015). The shifting perception on antioxidants: the case of vitamin E and beta-carotene. Redox Biol, 4, 272-8.

Wach A,Pyrzyńska K, Biesaga M (2007). Quercetincontent in some food and herbal samples. Food Chem, 100, 699-704.

Xia Q, Wang ZY, Li HQ, et al (2012). Reversion of p-glycoprotein-mediated multidrug resistance in human leukemic cell line by diallyltrisulfide. Evid Based Complement Alternat Med, 2012, 719805.

Xu Y, Zhi F, Xu G, et al (2012). Overcoming multidrugresistance in vitro and in vivo using the novel P-glycoprotein inhibitor 1416. Bio Sci Rep, 32, 559-66.

Yang C, Wong IL, Jin WB, et al (2014). Modification of marine natural product ningalin B and SAR study lead to potent P-glycoprotein inhibitors. Mar Drugs, 12, 5209-21.

Ye MX, Zhao YL, Li Y, et al (2012). Curcumin reverses cis-platin resistance and promotes human lung adenocarcinoma A549/DDP cell apoptosis through HIF-1alpha and caspase-3 mechanisms. Phytomedicine, 19, 779-87.

Yoshida N, Takagi A, Kitazawa H, et al (2005). Inhibition of P-glycoprotein-mediated transport by extracts of and monoterpenoids contained in Zanthoxylifructus. Toxicol Appl Pharmacol, 209, 167-73.

Zeino M, Paulsen MS, Zehl M, et al (2015). Identification of new P-glycoprotein inhibitors derived from cardiotonic steroids. Biochem Pharmacol, 93, 11-24.

Zhang S, Morris ME (2003). Effects of the flavonoids biochanin A, morin, phloretin, and silymarin on P-glycoprotein-mediated transport. J Pharmacol Exp Ther, 304, 1258-67.

Zhang W, Han Y, Lim SL, et al (2009). Dietary regulation of P-gp function and expression. Expert Opin Drug Metab Toxicol, 5, 789-801.

Zhao BX, Sun YB, Wang SQ, et al (2013). Grape seed procyanidin reversal of $\mathrm{p}$-glycoprotein associated multi-drug resistance via down-regulation of NF-KB and MAPK/ERK mediated YB-1 activity in A2780/T cells. PLoS One, 8, 71071.

Zhou S, Lim LY, Chowbay B (2004). Herbal modulation of P-glycoprotein. Drug Metab Rev, 36, 57-104.

Zorofchian Moghadamtousi S, Karimian H, Khanabdali $\mathrm{R}$, et al (2014). Anticancer and antitumor potential of fucoidan and fucoxanthin, two main metabolites isolated from brown algae. Sci J, 768323 . 